\title{
Correlating Interfacial Structure and Magnetism in Fe-MgO Thin Films Using Electron Microscopy, Neutron Reflectometry, and Atom Probe Tomography
}

\author{
S.R. Spurgeon, ${ }^{*}$ C.R. Winkler, ${ }^{*}$ B.J. Kirby, ${ }^{* *}$ C.L. Johnson, ${ }^{*}$ D.N. Seidman, ${ }^{* * *}$ and M.L. Taheri* \\ * Department of Materials Science \& Engineering, Drexel University, Philadelphia, PA 19104 \\ ** National Institute of Standards \& Technology - Center for Neutron Research, Gaithersburg, MD \\ 20899 \\ *** Department of Materials Science \& Engineering, Northwestern University, Evanston, IL 60208
}

A new generation of high efficiency electric motor designs requires novel soft magnetic composite materials, comprised of insulated powder metals [1]. We have developed a multi-scale characterization methodology to study soft magnetic composites that can be applied to a myriad of magnetic systems. By correlating atomic structure and magnetic order, we will be able to precisely tailor processing conditions to achieve desired properties in magnetic materials.

Powder metals have the potential to replace lamination steels used in induction cores, offering better formability and improved high frequency response [2]. These powders must first be coated with an electrically-resistive, magnetically-permeable material that can withstand high compaction pressures and annealing in excess of $500{ }^{\circ} \mathrm{C}$. Oxide coatings, particularly $\mathrm{Fe}-\mathrm{MgO}$, have been shown to reduce the eddy current losses associated with the magnetization of these materials [3]. Using thin film deposition we can model and study the interface between the iron core and oxide shell.

We have deposited iron films of 5-100 nm thickness on $\mathrm{MgO}$ (100) substrates using electron beam deposition. The films were capped with a protective layer of Au to prevent oxidation. Magnetization depth profiles of the entire film were then measured using the NG1 neutron reflectometer at NIST. The interface was studied using high resolution transmission electron microscopy. Elemental maps of the iron-magnesium oxide interface were constructed using scanning transmission electron microscopy and energy dispersive x-ray spectroscopy. Local electrode atom probe needles were prepared by annular ion milling to aid study of interfacial roughness and diffusion.

Our results clearly illustrate the complex interplay between growth conditions, atomic structure, and magnetic order. Variation in heat treatment and annealing can influence the formation of islands or uniform films (Fig. 1). While the films appear to have grown epitaxially, we note the presence of a magnetic dead layer near the Fe-MgO interface (Fig. 2). The saturation magnetization of the composite also differs markedly from that of bulk iron, hinting at possible interfacial strain or other defects. The methodology developed in this study will be invaluable in future analyses of magnetic interfaces and coatings. These results also point toward other potential coating systems, including Ni and $\mathrm{Zn}$ ferrites, which show promise for use in magnetic composites.

References

[1] K. Narasimhan, Metal Powder Report. 58 (2003) 12.

[2] L. Hultman et al., Advances in Powder Metallurgy and Particulate Matls. 14 (2002) 26.

[3] Y. Tanaka et al., World Congress on Powder Metallurgy and Particulate Matls. 10 (2008).

[4] This work is supported by NSF Grant DGE-0654313 and the Centralized Research Facilities at Drexel University's College of Engineering. 


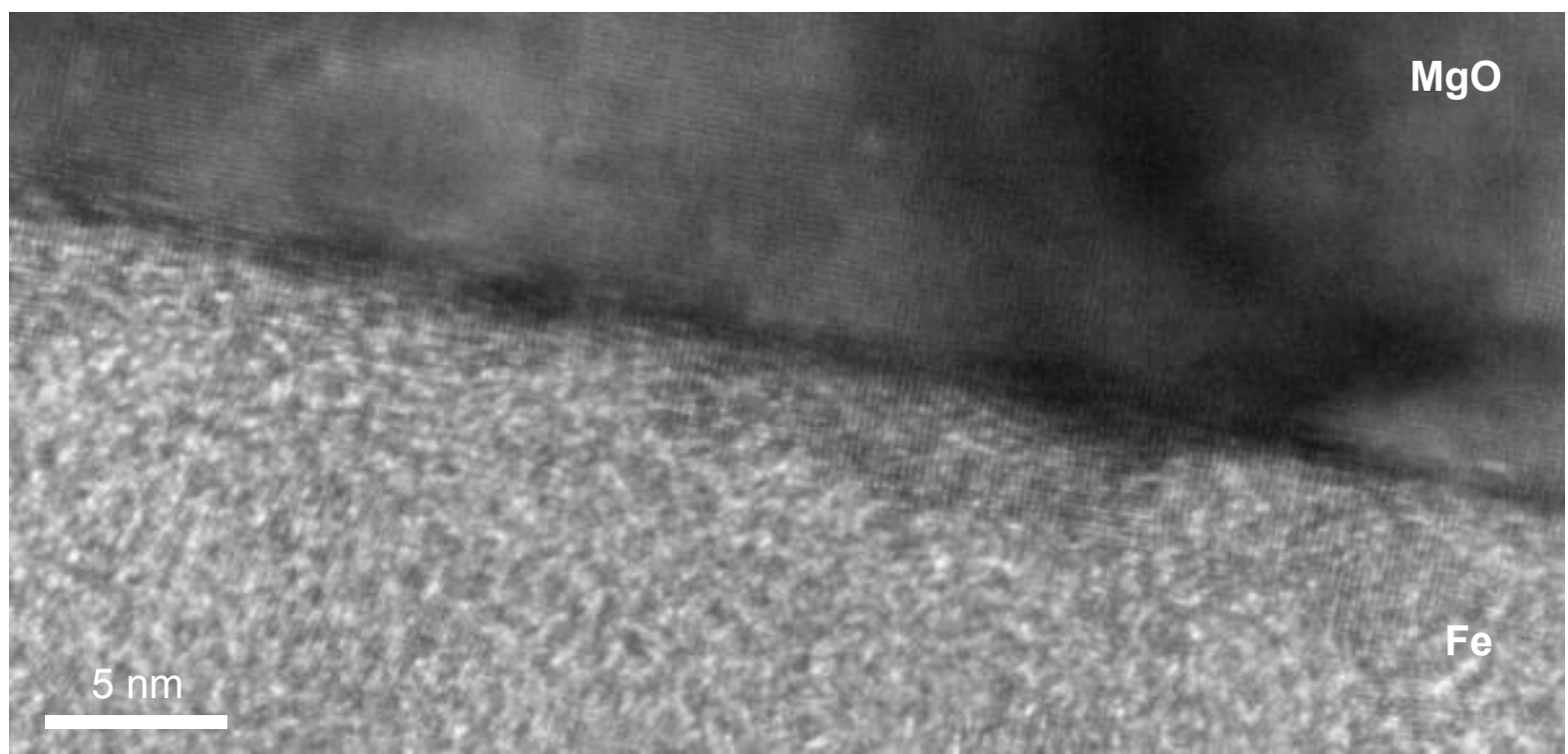

FIG. 1. HREM image illustrating the non-uniformity of the interface between Fe and $\mathrm{MgO}(100)$.

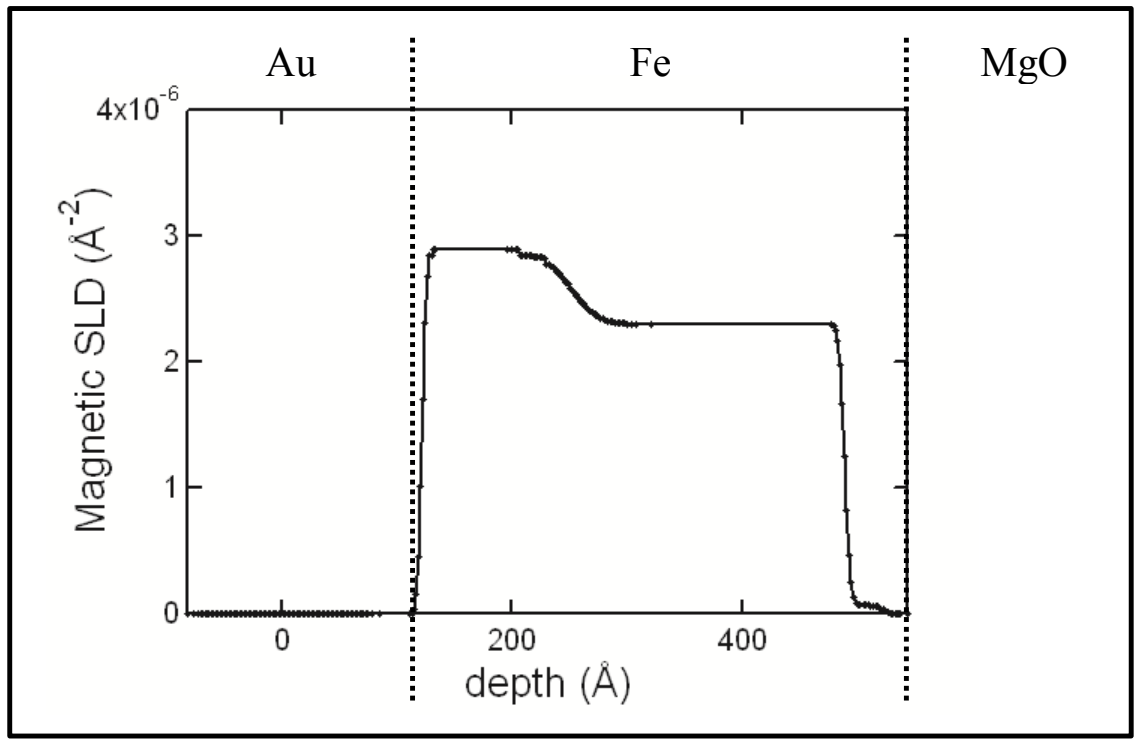

FIG. 2. Magnetization depth profile for a $10 \mathrm{~nm}$ Fe film on $\mathrm{MgO}$ (100) using electron beam deposition. This film was also capped with a $5 \mathrm{~nm}$ protective layer of Au to retard oxidation. The profile was calculated from polarized neutron reflectometry and indicates the presence of a magnetic dead layer at the film-substrate interface. 